\title{
Diagrammatic Presentation for the Production of Gravitons and Supersymmetry
}

\author{
AC Tahan ${ }^{1}$ \\ ${ }^{1}$ PO Box 391987, Cambridge MA 02139, USA \\ Correspondence: AC Tahan, PO Box 391987, Cambridge MA 02139, USA. E-mail: actahan2@yahoo.com
}

Received: July 2, 2012

Accepted: August 27, 2012

Online Published: August 31, 2012

doi:10.5539/mas.v6n9p76

URL: http://dx.doi.org/10.5539/mas.v6n9p76

\begin{abstract}
String experimentation has begun thanks to a novel laboratory technique that permitted the visualization of a D-brane (Tahan, 2011). Observations in experiments suggested that gravitons and related superparticles emerged due to the method. This manuscript describes the innovative technique diagrammatically. The method is shown to be useful for making particle predictions and for experimentation, particularly permitting nuclear and particle physics work including studies beyond the Standard Model to be performed on the lab bench.
\end{abstract}

Keywords: Graviton, graviphoton, graviscalar (radion), strings, D-brane

\section{Introduction}

Directing extremely low frequency radio waves with a specific amplitude $(\approx 2 \mathrm{~Hz}(2.000-2.012 \mathrm{~Hz})$ and $\mathrm{V}_{\mathrm{p}-\mathrm{p}}=4.312-4.437$, predominately 4.375) toward Hydrogen, sourced from typically $20 \mathrm{~mL}$ highly concentrated sulfuric acid (e.g. Duda Diesel sulfuric acid (98\% concd.) or 96\% concd. Mallinckrodt Analytical Reagent, ACS), in a static magnetic field (approximately $2000 \mathrm{Gs)} \mathrm{with} \mathrm{a} \mathrm{laser} \mathrm{(Quartet} \mathrm{Standard} \mathrm{Laser} \mathrm{Pointer)} \mathrm{directed} \mathrm{on} \mathrm{the}$ graphite tube (Crucible, Saed/Manfredi G40, 1.5"OD x 1.25"ID x 3.75"DP) holding the acid resulted in the appearance of a D-brane (Tahan, 2011) that resembled theorists' predictions (Thorlacius, 1998). Specifically, strings were understood to exist that supported a novel description of how gravitons and supersymmetry could emerge through string excitation. The method can be basically explained as placing a Hydrogen source in a magnetic field and coupling a distinct extremely low frequency to it.

The initial hypothesis based on principles of string theory that allowed for the conclusions in Figure 1 was that the underlying structure of Hydrogen (protons) involves strings, particularly gluon field strings, with tension that can be manipulated with a magnetic field and consequently forced to increase in vibration with electromagnetic energy of the correct frequency and amplitude based on the altered tension due to the magnetic field. Though the process can be considered a low energy method since it begins with only $2 \mathrm{~Hz}$ at a specific amplitude, the energy affecting the Hydrogen structure grows gradually from increasing string vibrations due to the $2 \mathrm{~Hz}$. In other words, a high energy is supplied from the structure itself. The energy eventually reaches the theoretically predicted level to allow for the indicated symmetry breaking of Figure 1 and related particles, as suggested with the reactions in section 3. Thus, a definition for energy can be visualized to be the string, and increasing vibration means a growing energy. Accordingly, this manuscript is a first explanation of the practical use of strings to produce gravitons and superparticles. An objective is to show through Figure 1 that the method can be used for predicting and experimentation, the figure having been based on experiments. The set-up that produced the figure can test new predictions inexpensively repeatedly and quickly.

\section{Background}

The source of the Hydrogen should be understood to have been the concentrated sulfuric acid. In Figure 1, neutron and graviton appearances are thought to have occurred near or at the same time--concluded based on Germanium (Ge) detector (Canberra 71255E, coarse gain 5) readings for neutron events related to isotope productions, the set-up without the laser having been placed in front of the detector. The origin of the neutron is considered to have been electron capture in Hydrogen. The gravitons and related bodies resulted from a symmetry breaking. The area labeled Spontaneous symmetry breaking/Unification in Figure 1 can be considered a region of unification of the forces of Nature but should not be confused with the grand unification at the 
beginning of the Universe. In the diagram, relatively high temperature is not necessary because of spontaneous symmetry breaking, including the evidence for strings and supersymmetry.

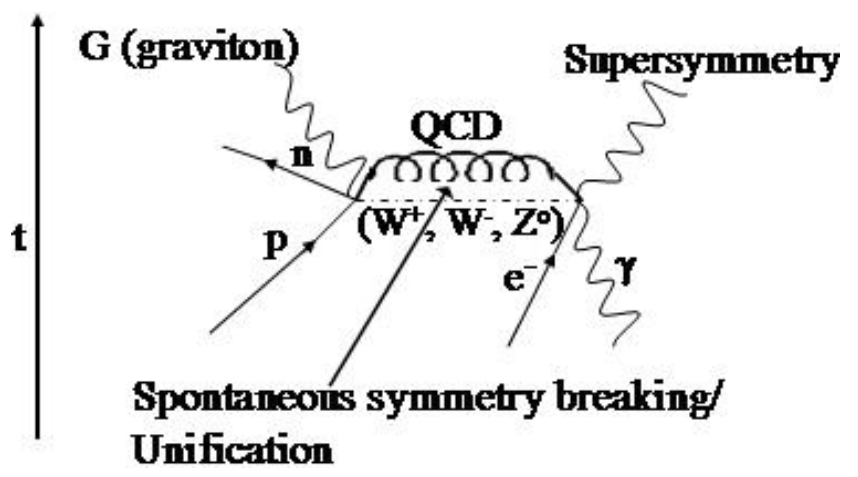

Figure 1. Feynman diagram of the process

The diagram illustrates the electron capture in Hydrogen (p) that led to the graviton and supersymmetry observations in trials: e.g. spacetime bending (Tahan, 2011). Though not in the diagram, an electron neutrino should be understood also to have resulted from the electron capture. The diagram should not be confusing if appreciating the objective to be an attempt to show an area of symmetry breaking in relation to the forces. For example, the time for QCD and the weak bosons is the same. Ideally the figure should be used with descriptions in the manuscript to understand fully the process.

A resulting electron neutrino was left-out of Figure 1 to reduce clutter since the particle was thought would be understood as part of the process of electron capture, thereby not needed to be shown. Figure 1 illustrates the most important steps and particularly the novel results. An alternative consideration to understand the progression would be to focus solely on the proton, which would still allow for photon interactions with strings to be imagined.

The bending of spacetime that created observations that were described as pushing and pulling influences in trials, concluded having been due to graviphotons and gravitons respectively (Tahan, 2011), was periodic thereby suggesting that energy increases were gradual. Thus, monitoring of progression toward the pushing and pulling influences can occur by accepting consistent energy levels or transitions, possibly also in consideration of string tension, that would be helpful particularly for advancing to certain points. For instance, a specific particle may be detected to emerge when a string vibration reaches a particular level, rather than needing to continue for symmetry breaking. Therefore, string vibrations can be regulated for specific purposes that can provide savings, e.g. cost and time.

\section{Using Figure 1}

Figure 1 was the result of experimentation and can be thought a simplified description of graviton production, containing the most crucial events. Thus, the diagram should not be thought to violate conservation laws. The symmetry breaking is primarily a facet of the gluon or strong force string. Figure $1 \mathrm{can}$ be written in the form of a reaction that can be helpful to visualize better the process: proton (spin 1/2, charge +1 ), electron (spin 1/2, charge -1 ), photon (spin 1, charge 0 ), gluon (spin 1, charge 0 ), $\mathrm{W}^{-}$( $\operatorname{spin} 1$, charge -1$) \rightarrow$ neutron (spin 1/2, charge 0 ), graviton (spin 2, charge 0 ), electron neutrino (spin 1/2, charge 0 ), graviphoton (spin 1 , charge 0 ), graviscalar or radion (spin 0 , charge 0 ).

The reaction is Figure 1; the components on the left side led to the right side results. The reaction need not be altered if wanting a simple explanation for graviton emergence. The reaction is useful to understand other components that may have been involved in the progression to gravitons, particularly if looking at the reaction in regards to conservation laws. Consideration of conservation laws can allow other bodies to be added to describe better the process, including for mass but to a limited extent. In other words, from experimentation the graviphoton was understood to be massive, observed with the pushing influence having a limited range. Accordingly, a source for the mass must exist on the left side of the reaction. Considering also that the graviscalar is massive based on observations in experiments and the predicted characteristics of the body in the reaction, a mass imbalance must exist. 
A massive particle that could be connected to a symmetry breaking is required on the left side of the reaction that would provide mass to the superpartners. Considering spin in the reaction, the body would have to be a spin 0 particle. The suggestion of a spin 0 body being in the reaction on the left side made the Higgs boson a possibility--unless other massive, spin 0 bodies may be candidates--since the Higgs mechanism is often connected to superparticles in the literature, including the graviscalar and graviphoton (Fackler \& Vân, 1989).

The reaction is atypical considering the placement of the weak interaction boson, photon, and gluon on the left side. The bosons were part of the process leading to the symmetry breaking that allowed for the graviton. The forces were involved or affected at different points in the process, including at symmetry breaking. Particularly when considering that the progression to the graviton involved strings, the three weak interaction bosons are listed in the diagram also as a consideration that the bosons may have participated in other events during the course of graviton production: e.g. possibly produced particles as neutrinos interacting with bodies thereby causing a boson to be involved.

The photon was the $2 \mathrm{~Hz}$ that affected the QCD strings (gluons), and the $\mathrm{W}^{-}$was involved in the neutron production, which originally was accepted as an extra result of the Figure 1 technique since focus had been only on the gluon field strings or gluon field excitation. By that, a gradual instability produced with the $2 \mathrm{~Hz}$ at a specific amplitude was due to the frequency directly affecting strings of the Hydrogen. Production of the neutron was thought supplemental because of the presence of the electron. The instability and increasing vibrations of the gluon field strings being equivalent to a growing energy (Scherk \& Schwarz, 1974) that was equal to gaining mass (Einstein, 1905) led to thought of the importance of the string body electron entering the nucleus. Specifically, being a string body the electron too was increasing in energy due to the resonance frequency--apart from an energy gain from the current or electric field resulting from three alligator clips, providing the $2 \mathrm{~Hz}$ from a function generator, being attached to the graphite tube. The electron eventually gained enough energy to tunnel through the potential barrier that separated it from the nucleus allowing for electron capture. And after further considering balancing the Figure 1 reaction, due to involved bosons, production of the neutron by electron capture was accepted important for graviton production involving symmetry breaking. Without the bosons on the left side, the reaction would violate conservation laws.

When understanding that the masses of the graviphoton and the graviscalar would be acquired from the symmetry breaking (Goldman, Hughes, \& Nieto, 1986), the possibility of the Higgs boson on the left side of the reaction, and the involvement of strings in the process to produce gravitons, the gluon in the reaction was concluded could be described eventually as a composite, e.g. a glueball or simply gluon fusion. Imagining the formation of the composite as the proton dissociating due to the string vibration amount reaching or passing a maximum (Franck, 1926; Condon, 1926) can allow for the strings to combine for the composite. Gluon fusion has been described as a path to the Higgs boson (Bezares-Roder \& Nandan, 2008). Also, the radion has been predicted can be the result of gluon fusion (Chaichian, Datta, Huitu, \& Yu, 2002), many publications could have been cited. The gluon fusion is different than typically discussed since it is due to related string vibrations. Hence, the three gluons of the proton can be considered on the left side of the reaction.

A composite weak interaction boson was thought a possible result of the symmetry breaking. Though the particle has not been discounted, a composite boson based on predictions (Fritzsch, 2010) seemed would violate conservation laws in the above reaction. Again the reaction is the simplest form of how to produce a graviton and supersymmetry. Other particles due to the technique for Figure 1 may have been produced on the right side of the reaction that would allow for different or additional considerations for the left side. Specifically, bodies can be added to the reaction as the particles are discovered without the additions altering the primary components involved in graviton production, i.e. Figure 1, which was consistent due to the description of the set-up in the introduction section.

Though a specific composite weak interaction boson can be introduced, need for a new particle when accepting the Glashow-Weinberg-Salam model in the reaction--symmetry breaking in relation to the Higgs boson, the photon, and the $\mathrm{SU}(2)$ gauge theory of the weak interaction--is not needed. The $\mathrm{Z}$ boson would appear as a mixture of the neutral isospin $\mathrm{SU}(2)$ and the hypercharge U(1) gauge fields (Salam \& Ward, 1964; Weinberg, 1967; Glashow, 1961). Thus, the $Z$ boson was added to the left side of the reaction after understanding that the boson, particularly when remembering the symmetry breaking, could couple to the Higgs boson (Bezares-Roder \& Nandan, 2008).

Further imbalance of conservation laws occurred after inclusion of the three gluons and the $\mathrm{Z}$ boson. Placing the Figure 1 set-up--without the laser--in front of a Ge detector led to a recording of photons from the tube, including between forty and fifty minutes when the largest pulling observations, graviton-related effects, had 
occurred. Thus, photons on the right side were thought could balance the reaction. The $\mathrm{Z}$ boson decaying to a photon and graviton has been predicted (Nieves \& Pal, 2005). Accordingly, a photon was added to the right side. Observations of photons at the times when the largest pulling influences had been observed indicated the photons occurred near the time of symmetry breaking, suggesting the decay to photons of a particle involved in symmetry breaking. The Higgs boson possibly decaying to two photons has been reported (Gastmans, Wu, \& $\mathrm{Wu}, 2011$ ), which led to two additional photons being placed on the right side of the reaction. Continuing to consider the conservation laws, the parity on the left side was not balanced after the addition of the photons.

Ge detector readings in usual one hour trials were observed consistently enough between forty and fifty minutes, including at other times when pulling and pushing influences had been recorded in experiments, to accept that the readings were related to events due to the set-up. The observations could not be dismissed easily as due to an alternate source as the environment particularly after performing various control trials involving varying the set-up, including running the Ge detector alone for background comparisons.

An $\approx 1.6 \mathrm{MeV}$ reading was typically recorded with the Ge detector between forty and fifty minutes. Though counts were low and accordingly would not typically have been stated in this manuscript, the observation is mentioned for the purpose of discussion since a $1.6 \mathrm{MeV}$ reading has been suggested could represent axion production (Balantekin et al., 1985; Tsai, 1986), which would suggest a relation to graviton creation due to U(1) symmetry breaking (Weinberg, 1978; Wilczek, 1978) involving the Higgs field (Schäfer, 1989). The magnetic field and the cooling of the Ge crystal could have contributed to detections (Sikivie, 1983). Scientists have reiterated that axions could be evidence for string theory and have suggested that rotating black holes could release energy and particles as axions (Arvanitaki et al., 2010). But the low counts could indicate that the approximately $1.6 \mathrm{MeV}$ reading was not related to the axion or if the recordings were due to an event, the reading may have been from a different process in the tube.

Initially low counts were explained by being thought to represent singular events rather than a large supply of photons as from an isotope source. According to a representative from the manufacturer, the detector was not designed for detections near or above $1 \mathrm{MeV}$; low efficiency exists at high energies for the detector. However, the representative indicated that low counts should not be dismissed, particularly after comparison to data from the environment and control trials. Still, running the set-up with a more sensitive detector may be helpful. Axions would resolve the strong CP problem, contributing to the Higgs mechanism that made placement on the left side of the reaction reasonable, and could be evidence for dark matter (Kuster, Raffelt, \& Beltrán, 2008).

If accepting that experimentation exposed supergravity, the gravitino must be placed on the right side of the reaction. Considering the gravitino spin and the gluons on the left side, gluinos can be added to the left side to balance the reaction. Consequently the reaction can be written more completely: gluon, gluon, gluon, gluino, gluino, gluino, photon, proton, electron, $\mathrm{W}^{-}$boson, Higgs boson, $\mathrm{Z}$ boson, axion, dilaton $\rightarrow$ graviton, gravitino, neutron, electron neutrino, graviscalar, graviphoton, photon, photon, photon, sneutrino, selectron, saxion.

The dilaton, sneutrino, selectron, and saxion were additionally added to the reaction to balance it related to conservation laws. Placement of the dilaton was considered when remembering the D-brane that suggested extra dimensions, especially that the dilaton appears in Kaluza-Klein theory related to the compactification of extra dimensions. Also, when considering that the axion could be on the left side of the reaction, that the dilaton has been predicted to combine with the axion to form a scalar field that is always associated with gravity, and that it appears in all modern unified theories including the superstring that are based on higher-dimensional physics (Alvarenga, Batista, \& Fabris, 2005; Cho \& Kim, 2009), inclusion of the dilaton was thought logical.

In certain theories, graviscalar (radion) or dilaton is synonymous for the same particle. In the more detailed reaction above, the bodies are different. The graviscalar is a massive, neutral particle (Pollard, 1983; Barger \& Ishida, 2011; Adelberger, Heckel, Stubbs, \& Su, 1991; Maartens, 2004; Gennai, 2003), and the dilaton is a light, neutral body accompanying symmetry breaking (Okun, 1991). Yet, the graviscalar can be thought related to the dilaton if the dilaton is on the left side of the reaction. Again this paper is based on experimentation involving the set-up described in the introduction. Presenting new theoretical physics is not the intention, unless new theory can be understood from experiments. The dilaton was placed on the left side of the reaction to balance it, as having been explained. But further experimentation with the technique involving proper detectors may find a different particle to be placed on the left side. The sneutrino, selectron, and saxion were added to the right side after considering supergravity and supersymmetry in regards to the axion, electron, and the production of a neutrino and since the spin 0 , charges, and parity of the bodies balance the reaction.

The initial reaction represented the figure and demonstrated the usefulness of Figure 1, particularly to understand the particles in the process of releasing gravitons. Different particles may be inserted in the basic reaction, 
including replacing bodies in the more detailed reaction particularly if one body as the axion is found to be a different particle. The detailed reaction is incomplete and discussion could continue possibly to include other bodies--e.g. neutralino, dilatino, etc--when considering the present particles. Also, the more detailed reaction can be useful to help detect particles, e.g. superparticles, that may be due to the decay of a specific body including a body not in the reaction. But more additions would not present better the usefulness of Figure 1 and would make this manuscript more of a theoretical physics paper than was intended. Use of the technique, a relatively easier method to produce particles, with an appropriate detector or using the set-up in conjunction with a different method would be the best manner for proceeding with the reaction of Figure 1.

The Higgs boson in the reaction suggested that a mass prediction was possible. The calculation must be understood was based on particle masses that resulted from the Higgs mechanism, specifically the mass of the Higgs boson, or that were significantly involved in the emergence of the boson. Therefore, possible masses of bodies particularly on the left side of the reaction, e.g. the gluino (Alwall, Le, Lisanti, \& Wacker, 2008), are not included but may be incorporated in future publications if detector studies find that the particles are more related to the emergence of the boson.

The main mass components on the right side of the initial reaction are the radion and graviphoton. Certain bodies in the detailed reaction were not considered for a calculation having been thought would not be massive enough to influence significantly a prediction, e.g. the axion and dilaton having been accepted as light particles (Okun, 1991). The gravitino was considered insufficiently massive to alter considerably a calculation; thus, it was not included. Still, gravitino mass varies depending on the prediction model (Ratz, 2006) and could be included in a calculation depending on results from future studies.

The $\mathrm{Z}$ boson mass is part of the calculation since it could have been placed in the initial reaction, as the electron neutrino could have been inserted in Figure 1, and its mass is due to the Higgs mechanism. Though not due to the Higgs mechanism, the composite gluon or glueball mass is included in the calculation since the likely emergence of the gluon fusion was considered would be a significant step in the eventual appearance of the Higgs boson (Bentvelsen, Laenen, \& Motylinski, 2005), if having placed the boson in the detailed reaction was correct. The excitation of the gluon field strings and resulting growing energy (Scherk \& Schwarz, 1974) and increasing mass (Einstein, 1905) led to the gluon fusion and symmetry breaking. Accordingly, possible masses for the glueball were considered for inclusion in a Higgs boson mass prediction. The gluon fusion is written uniquely in the detailed reaction to balance it, but documenting the gluon fusion can be as typically written in literature--gg--since the gluon fusion was thought to result in the glueball. Again stimulation of the gluon field strings due to the novel technique may have allowed for different particles to emerge, including a body with a mass similar to predictions for the glueball.

The calculation considered the left and right sides of the reaction as separate groups: right side - left side=Higgs boson mass. The graviscalar mass $300 \mathrm{GeV}$ is the primary uncertainty. A significant change in the mass would alter the calculation. But the radion mass was predicted based on a Higgs boson mass of $125 \mathrm{GeV}$ (Gennai, 2003), which is close to the calculated mass: $125.81 \mathrm{GeV} \approx$ (graviscalar mass + graviphoton mass)-( $\mathrm{Z}$ boson mass $+\mathrm{W}^{-}$ boson mass+glueball mass). The glueball mass, instantons having been suggested contribute (Mathieu, Kochelev, \& Vento, 2009), included in the calculation was the heaviest of the predicted lightest masses $(2.59 \mathrm{GeV})$ for the composite body, due to consideration of the detailed reaction involving the three gluons. But a different glueball mass may be more suitable to include, or a prediction range due to including each of the lightest glueball masses $(1.73 \mathrm{GeV}, 2.4 \mathrm{GeV}, 2.59 \mathrm{GeV})$ (Morningstar \& Peardon, 1999; Mathieu, Kochelev, \& Vento, 2009) perhaps is more appropriate. By that, the mass using the detailed reaction can be adjusted based on the most accepted values for involved particles, particularly considering that bodies may be removed or inserted in the reaction. Other particles or superparticles may be added to a calculation if detected in future experiments, including reusing the reactions when new particles are added from experimentation to forecast novel bodies. Also, a detector reading for the Higgs boson mass may lead to additions to the detailed reaction, possibly including predictions of new particles, if the above calculation is significantly different.

The masses for the weak interaction bosons were the typical masses presented in literature, and the graviphoton mass was $1.685206718 \times 10^{-29} \mathrm{GeV}$ (Argyris \& Ciubotariu, 1997). Because the pushing influence observed in experiments and thought due to the graviphoton had a limited range, if accepting a prediction for the graviphoton range to be $10^{15} \mathrm{~cm}$ (Argyris \& Ciubotariu, 1997) the graviton and possibly graviscalar limited the affect of the graviphoton in trials. Decay to two photons as in the detailed reaction has been predicted for the Higgs boson if it is in a range of masses that includes $125.81 \mathrm{GeV}$ (Chaichian, Datta, Huitu, \& Yu, 2002). Though the mass remains a possibility based on published conclusions from detector groups (Nisati, 2011; Sharma, 2011; Verzocchi, 2011; Nakamura et al., 2010), it was only presented to show further the usefulness of Figure 1 and is 
not a definite measure; the mass is simply a demonstration. Otherwise, approximately $125.81 \mathrm{GeV}$ could have been presented formerly sooner since it is based on results from the technique described in the introduction section that has been in use for over a decade. Again the calculation involves the masses of bodies in the detailed reaction and would be different depending on what values are used for certain particles.

Figure 1 and the initial reaction, apart from trials that led to the drawing of Figure 1, simply suggested that a spin 0 , neutral charged, massive body was involved in graviton productions due to the innovative set-up. If the Higgs boson exists, the only means to know the mass is through the direct searches underway. The intention of this manuscript was to present conclusions from experiments using the innovative technique mentioned in the introduction and to demonstrate the utility of the results for predictions that could lead to additional research. Again this paper was not to be a theoretical physics work, not intended to be a reference for a prediction of the Higgs boson mass. The usefulness of Figure 1 was to be demonstrated, and again adding to the reactions can occur through further experimentation.

\section{Conclusions}

This manuscript showed the utility of Figure 1, representing the landmark method described in the introduction section for experimentation and predictions. The minimal description of the technique should not be thought missing components; the specification is simple and allows for easily conductible experiments. Figure 1 being based on experiments presents a first explanation for how to produce gravitons and superparticles, previously the bodies not being known to exist apart from in theoretical discussions. The novel set-up can be used independently for experiments and predictions or in conjunction with facilities that are more accepted in the scientific community as standard techniques for studies.

This manuscript being originally part of a larger body of work in progress, initially no intention existing to write it separately, was submitted firstly partly as a matter of concern, particularly that superparticles have not been detected at synchrotrons while celebrations of the gradual confirmations of the Higgs boson seem to be quieting the fact. But for decades the Higgs boson has been considered must exist, synchrotron detections only having strengthened the belief of existence. Obviously the concern emerged due to knowledge of the work that allowed for this manuscript; the quiet regarding superparticles might be less if the innovative technique and related results were more discussed.

If a detected Higgs boson mass at synchrotrons is found between $125-126 \mathrm{GeV}$, a reading near the same level as the calculation of this manuscript, no detections of supersymmetry by the end of 2012 would support that innovation is required to conduct science beyond the Standard Model, though collisions at larger energies eventually could allow for the reporting of superparticles. The significantly more inexpensive and facile nature for superparticle studies with the method of this manuscript has allowed for repeated, periodic superparticle-related observations in approximately one hour trials, at least of pushing influences due to graviphotons, and has permitted the direct visualization of strings including graviton-related effects (Tahan, 2011). Consequently, the technique for Figure 1 that sourced energy primarily from the proton by string vibrations may eventually be accepted universally to be a useful method for studies beyond the Standard Model, apart from Figure 1 serving as a tool for detecting superparticles.

\section{References}

Adelberger, E. G., Heckel, B. R., Stubbs, C. W., \& Su, Y. (1991). Does Antimatter Fall with the Same Acceleration as Ordinary Matter? Phys. Rev. Lett., 66, 850-853. http://dx.doi.org/10.1103/PhysRevLett.66.850

Alvarenga, F. G., Batista, A. B., \& Fabris, J. C. (2005). Does Quantum Cosmology Predict a Constant Dilatonic Field? Int. J. Mod. Phys., 14D, 291-308. http://dx.doi.org/10.1142/S0218271805005955

Alwall, J., Le, M-P., Lisanti, M., \& Wacker, J. G. (2008). Searching for gluinos at the Tevatron. SLAC-PUB-13149.

Argyris, J., \& Ciubotariu, C. (1997). Massive Gravitons in General Relativity. Aust. J. Phys., 50, 879-891. http://dx.doi.org/10.1071/P97002

Arvanitaki, A., Dimopoulos, S., Dubovsky, S., Nemanja, K., \& March-Russell, J. (2010). String axiverse. Phys. Rev., 81D, 123530. http://dx.doi.org/10.1103/PhysRevD.81.123530

Balantekin, A. B., Bottcher, C., \& Strayer, M. R. (1985). Production of new particles in heavy-ion collisions. Phys. Rev. Lett., 55, 461-464. http://dx.doi.org/10.1103/PhysRevLett.55.461

Barger, V., \& Ishida, M. (2011). Randall-Sundrum Reality at the LHC. arXiv: 1110.6452v2 [hep-ph], 1-14. 
Bentvelsen, S., Laenen, E., \& Motylinski, P. (2005). Higgs production through gluon fusion at leading order. NIKHEF, 2005-007.

Bezares-Roder, N. M., \& Nandan, H. (2008). Spontaneous Symmetry Breakdown and Critical Perspectives of Higgs Mechanism. Indian J. Phys., 82, 69-93.

Chaichian, M., Datta, A., Huitu, K., \& Yu, Z. (2002). Radion and Higgs mixing at the LHC. Phys. Lett., 524B, 161-169. http://dx.doi.org/10.1016/S0370-2693(01)01378-8

Cho, Y. M., \& Kim, J. H. (2009). Dilatonic dark matter and its experimental detection. Phys. Rev., 79D, 023504. http://dx.doi.org/10.1103/PhysRevD.79.023504

Condon, E. (1926). A theory of intensity distribution in band systems. Phys. Rev., 28, 1182-1201. http://dx.doi.org/10.1103/PhysRev.28.1182

Einstein, A. (1905). Ist die Trägheit eines Körpers von seinem Energieinhalt abhängig? Annalen der Physik, 18, 639-643. http://dx.doi.org/10.1002/andp.19053231314

Fackler, O., \& Vân, J. T. T. (1989). Tests of fundamental laws in physics. Editions Frontières, Gif-sur-Yvette Cedex, France.

Franck, J. (1926). Elementary processes of photochemical reactions. Trans. Faraday Soc., $21,536-542$. http://dx.doi.org/10.1039/tf9262100536

Fritzsch, H. (2010). Composite Weak Bosons. arXiv: 1010.1428v2 [hep-ph], 1-8.

Gastmans, R., Wu, S. L., \& Wu, T. T. (2011). Higgs Decay into Two Photons, Revisited. CERN-PH-TH/2011-201, arXiv: 1108.5872v1 [hep-ph], 1-20.

Gennai, S. (2003). Search of a graviscalar particle of the Randall-Sundrum model with the CMS experiment at LHC. CERN-THESIS-2009-091, 1-185.

Glashow, S. L. (1961). Partial-symmetries of weak interactions. Nucl. Phys., 22, 579-588. http://dx.doi.org/10.1016/0029-5582(61)90469-2

Goldman, T., Hughes, R. J., \& Nieto, M. M. (1986). Experimental evidence for quantum gravity? Phys. Lett., 171B, 217-222. http://dx.doi.org/10.1016/0370-2693(86)91535-2

Kuster, M., Raffelt, G., \& Beltrán, B. (2008). Axions: theory, cosmology, and experimental searches. Berlin: Springer-Verlag.

Maartens, R. (2004). Brane-world gravity. Living Rev. Rel., 7, 1-99.

Mathieu, V., Kochelev, N., \& Vento, V. (2009). The physics of glueballs. Int. J. Mod. Phys., 18E, 1-49. http://dx.doi.org/10.1142/S0218301309012124

Morningstar, C. J., \& Peardon, M. (1999). Glueball spectrum from an anisotropic lattice study. Phys. Rev., 60D, 034509. http://dx.doi.org/10.1103/PhysRevD.60.034509

Nakamura, K. (Particle Data Group) (2010). Review of Particle Physics. J. Phys. G: Nucl. Part. Phys., 37, 075021. http://dx.doi.org/10.1088/0954-3899/37/7A/075021

Nieves, J. F., \& Pal, P. B. (2005). Gravitational decay of the Z-boson. Phys. Rev., 72D, 093006. http://dx.doi.org/10.1103/PhysRevD.72.093006

Nisati, A. (2011). Talk at the XXV International Symposium on Lepton Photon Interactions at High Energies. Mumbai, India.

Okun, L. B. (1991). The relation of particles. Singapore: World Scientific Publishing.

Pollard, D. (1983). Antigravity and classical solutions of five-dimensional Kaluza-Klein theory. J. Phys. A: Math. Gen., 16, 565-574. http://dx.doi.org/10.1088/0305-4470/16/3/015

Ratz, M. (2006). Theoretical aspects of gravitinos. Mini-workshop on Superweakly Interacting Dark Matter, DESY.

Salam, A., \& Ward, J. C. (1964). Electromagnetic and weak interactions. Phys. Lett., 13, $168-171$. http://dx.doi.org/10.1016/0031-9163(64)90711-5

Schäfer, A. (1989). New particles in strong fields? J. Phys. G: Nucl. Phys., 15, $373-416$. http://dx.doi.org/10.1088/0954-3899/15/4/004 
Scherk, J., \& Schwarz, J. H. (1974). Dual models for non-hadrons. Nucl. Phys., 81B, 118-144. http://dx.doi.org/10.1016/0550-3213(74)90010-8

Sharma, V. (2011). Talk at the XXV International Symposium on Lepton Photon Interactions at High Energies. Mumbai, India.

Sikivie, P. (1983). Experimental Tests of the "Invisible" Axion. Phys. Rev. Lett., 51, 1415-1417. http://dx.doi.org/10.1103/PhysRevLett.51.1415

Tahan, A. C. (2011). Exposing strings in the laboratory with a novel technique. Appl. Phys. Res., 3(2), 39-51. http://dx.doi.org/10.5539/apr.v3n2p39

Thorlacius, L. (1998). Introduction to D-Branes. Nucl. Phys. Proc. Suppl., 61A, 86-98. http://dx.doi.org/10.1016/S0920-5632(97)00521-5

Tsai, Y. S. (1986). Axion bremsstrahlung by an electron beam. Phys. Rev., 34D, 1326-1331. http://dx.doi.org/10.1103/PhysRevD.34.1326

Verzocchi, M. (2011). Talk at the XXV International Symposium on Lepton Photon Interactions at High Energies. Mumbai, India.

Weinberg, S. (1967). A Model of Leptons. Phys. Rev. Lett., 19, 1264-1266. http://dx.doi.org/10.1103/PhysRevLett.19.1264

Weinberg, S. (1978). A New Light Boson? Phys. Rev. Lett., 40, 223-226. http://dx.doi.org/10.1103/PhysRevLett.40.223

Wilczek, F. (1978). Problem of Strong P and T Invariance in the Presence of Instantons. Phys. Rev. Lett., 40, 279-282. http://dx.doi.org/10.1103/PhysRevLett.40.279 